\title{
Density of Polyethylene Crystals Grown From Solution
}

\author{
Gordon M. Martin and Elio Passaglia \\ Institute for Materials Research, National Bureau of Standards, Washington, D.C.
}

(February 9, 1966)

\begin{abstract}
The density of Marlex 50 polyethylene crystals grown from a 0.75 percent solution in $p$-xylene at $70{ }^{\circ} \mathrm{C}$ was measured by a pycnometric method. Difficulties in making the measurements to the necessary accuracy are discussed. The values obtained were close to $0.98 \mathrm{~g} / \mathrm{cm}^{3}$ at $30{ }^{\circ} \mathrm{C}$ and significantly less than the crystallographic density of $1.00 \mathrm{~g} / \mathrm{cm}^{3}$. Several possible explanations of this density deficiency are mentioned.
\end{abstract}

Key Words: Crystals, density, polymer, p-xylene, polyethylene, pycnometer, density deficiency, weighing method.

\section{Introduction}

The measured density of a crystalline polymer is usually less than that calculated from the density of its unit cell. This reduced density has been attributed to the amorphous regions in a two phase amorphouscrystalline model or to defects and "mispacking" of folds in adjacent lamella in a chain-folded crystalline model. Crystals of polymers grown from dilute solution appear to have many of the characteristics of single crystals of nonpolymeric materials, and there has been special interest in their densities.

The density of polyethylene crystals grown from solution has been measured by a centrifuge method $[1,2],{ }^{1}$ a gradient tube method [1], and a pycnometer method [1,3]. Values around $0.97 \mathrm{~g} / \mathrm{cm}^{3}$ have been obtained by all these methods for crystals formed at $70{ }^{\circ} \mathrm{C}[1,2]$ as compared to the crystallographic density of nearly $1.00 \mathrm{~g} / \mathrm{cm} .^{3}$ However, values of about 1.00 $\mathrm{g} / \mathrm{cm}^{3}$ have been obtained by a pycnometer method for the density of the crystals [3].

Although the density gradient tube method is subject to some uncertainty [4], the centrifuge method, in which the density of a buoying liquid is adjusted to be equal to that of the crystals, appears to be as valid as pycnometric methods. It is clear that a discrepancy exists. The present work was undertaken to provide another pycnometric measurement of the density of polyethylene crystals.

\section{Experimental Procedure}

To prepare the crystals, Marlex $50^{2}$ polyethylene (with a weight average molecular weight of 130,000 ) was first dissolved in xylene at $145{ }^{\circ} \mathrm{C}$, allowed to crystallize at $80{ }^{\circ} \mathrm{C}$, and rinsed with xylene at $80{ }^{\circ} \mathrm{C}$ to remove low molecular weight fractions or other material. The polymer was dried under vacuum at room temperature. Enough of this treated polyethylene to make a 0.75 percent solution was dissolved in distilled $p$-xylene at $145{ }^{\circ} \mathrm{C}$ and allowed to crystallize for a week at $70^{\circ} \mathrm{C}$. Micrographs of some typical crystals are shown in figures 1 and 2 .

The pycnometers used, which had a nominal volume of $50 \mathrm{~cm}^{3}$, were made from Pyrex glass as illustrated in figure 3 . They were annealed and the standard taper joints were ground together by hand using fine emery in water. They were filled by a hypodermic syringe with a needle which was inserted through the capillary tube. The pycnometers could be emptied either by using the hypodermic syringe or by turning upside down and connecting to an aspirator. This was somewhat inconvenient, but the single, carefully fitted joint which could be dried before assembly reduced the rate of evaporation of an enclosed liquid.

The pycnometers were cleaned with a sulfuric acid and sodium dichromate solution, rinsed several times with distilled water, and dried overnight at $120{ }^{\circ} \mathrm{C}$. After cleaning they were always handled with forceps or rubber gloves.

${ }^{2} \mathrm{~A}$ commerical material is identified in this paper in order to adequately specify the experimental procedure. Such identification does not imply recommendation or endorse ment by the National Bureau of Standards. 

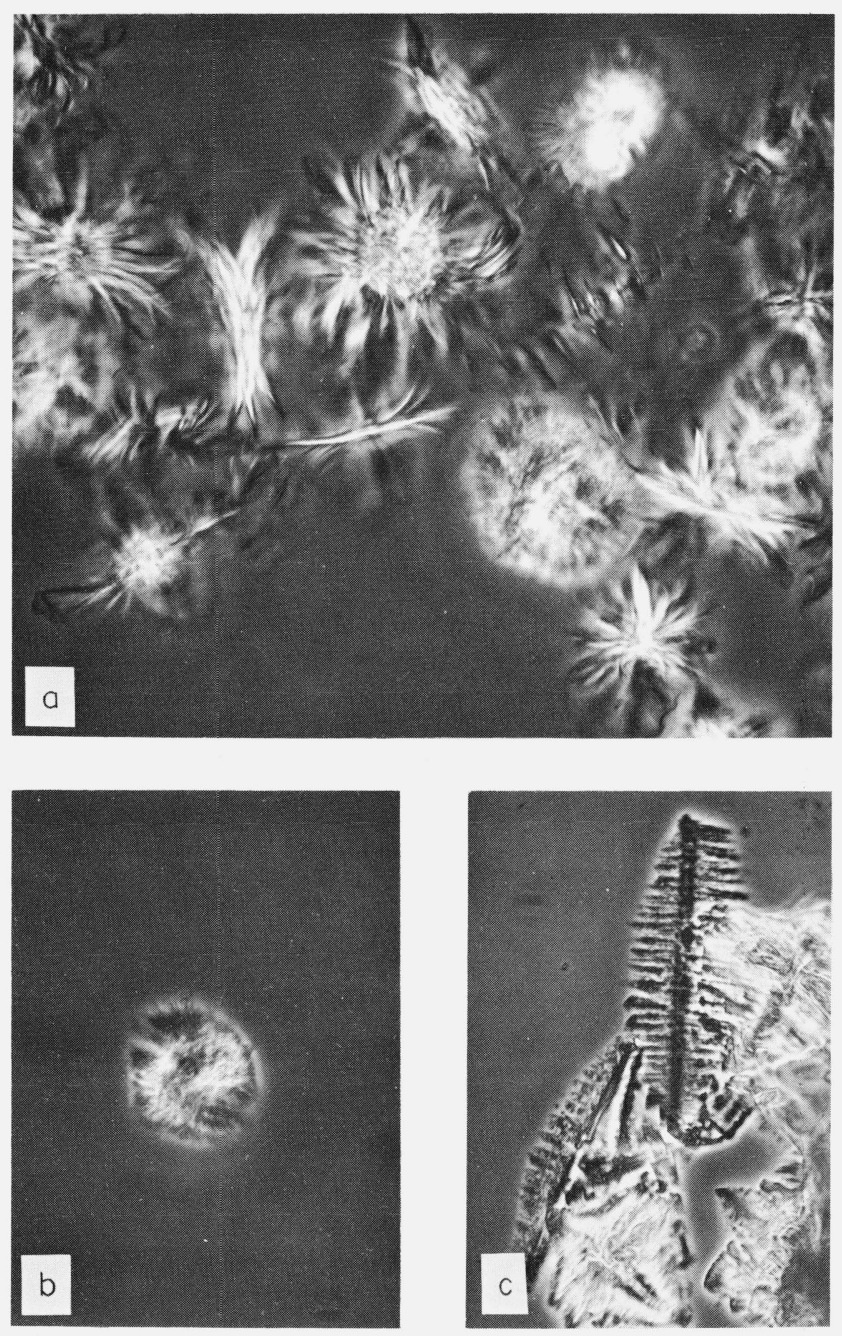

FigurE 1. Optical phase contrast micrographs of the type of crystal aggregates in the preparation; $\times 155$ (a) Dendrites and axialites in suspension in xylene. Note distinct splaying of layers in some crystals. (b) Axialite in suspension in xylene. (c) Dendrites after drying.

Weights were taken to the nearest $0.05 \mathrm{mg}$ on a Mettler single-pan balance. The temperature, barometric pressure, and relative humidity were measured at each weighing and the density of the air was calculated, so that the observed weights could be corrected for the net buoyant force of the atmosphere.

The pycnometers were filled at $30.15{ }^{\circ} \mathrm{C}$ in a water bath which was regulated within $\pm 0.01{ }^{\circ} \mathrm{C}$. The liquid was first adjusted above the line on the capillary tube; then small, rolled strips of lens tissue were dipped in it until the level was lowered almost to the line. The liquid was then allowed to evaporate until the meniscus just touched the line when observed through a jeweler's eyepiece.

After weighing empty and filled with boiled, distilled water, the pycnometers were weighed filled with distilled $p$-xylene which had been taken from the same flask as that used for preparing the crystals

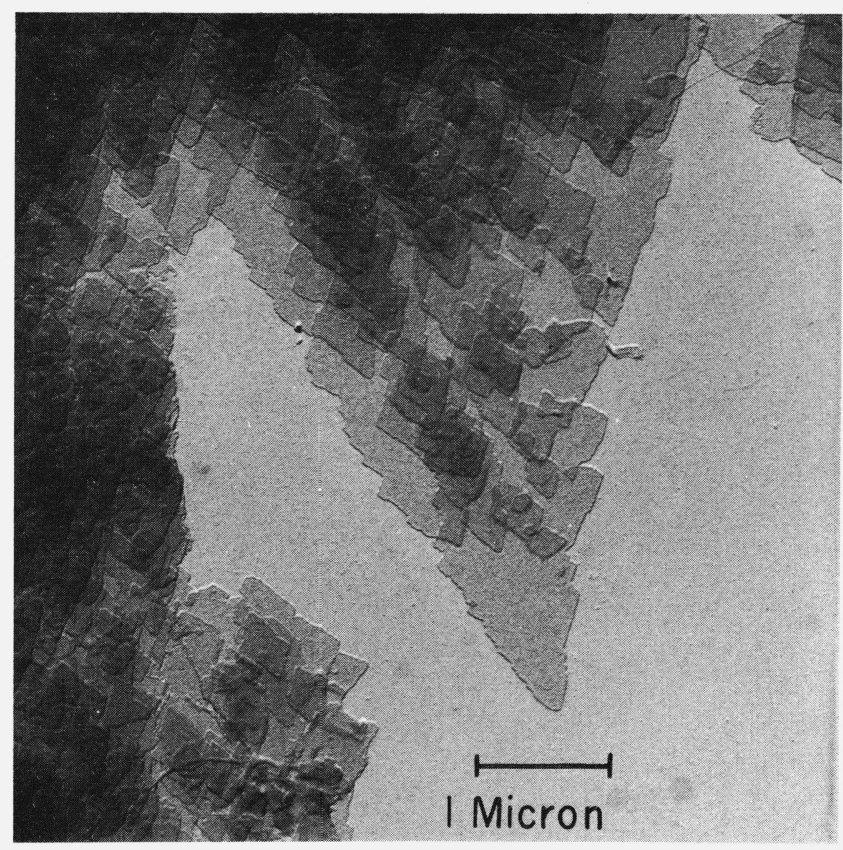

FIGURE 2. Electron micrograph of a portion of a dendrite similar to those shown in figure 1 (c).

and had been carried through exactly the same heat treatment prior to having been put in the pycnometers. The average of six determinations gave a density of $0.852241 \mathrm{~g} / \mathrm{cm}^{3}$ for the $p$-xylene at $30.15{ }^{\circ} \mathrm{C}$. The standard deviation of an individual result was 0.000003 $\mathrm{g} / \mathrm{cm}^{3}$.

In the final step in the procedure, after the pycnometer had been weighed filled with the mixture of $p$ xylene and polyethylene crystals, it was necessary to evaporate the liquid and weigh the pycnometer and dried crystals alone. This was done in a vacuum. However, if the pycnometer was placed immediately in the vacuum chamber, vapor bubbles could form and cause some of the liquid and crystals to splash

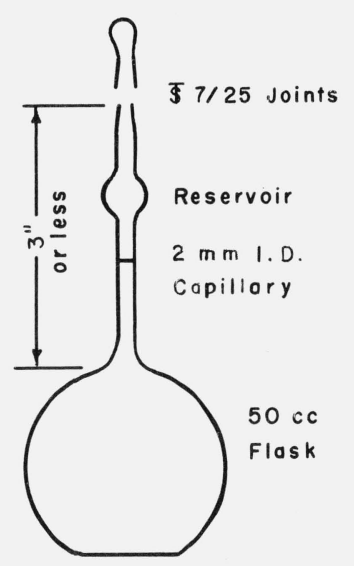

Figure 3. Pycnometer, Pyrex glass. 
out. To avoid this the pycnometer was heated between 35 and $40{ }^{\circ} \mathrm{C}$ for several days to evaporate some of the liquid and create a space below the capillary. The reservoir above the capillary prevented overflowing during the heating. After heating, the pycnometer was placed in a desiccator connected to a vacuum pump and the valve opened very gradually so that an hour or more was required to produce the final vacuum. Pumping at room temperature was continued for a day after the crystals appeared dry. The pycnometer was then placed in a vacuum oven at $100{ }^{\circ} \mathrm{C}$ for several days. No significant change in weight was observed after the first day in the vacuum oven.

After this treatment, it was assumed that there was not enough xylene left in the crystals to have an appreciable effect on their density. Although we know of no data on the retention of xylene when crystals of polyethylene are dried under our conditions, experiments with such crystals grown in tetrachloroethylene have shown that approximately 0.1 percent by weight of that solvent remained in the crystals when they were heated to $60{ }^{\circ} \mathrm{C}$ in a vacuum [5]. If this much xylene remained in our crystals, it would reduce the density by only $0.0002 \mathrm{~g} / \mathrm{cm}^{3}$, which is well within the experimental error from other sources. Moreover, in order for the density of the crystals to be lowered from the crystallographic value of 1.00 $\mathrm{g} / \mathrm{cm}^{3}$ to the observed value of $0.98 \mathrm{~g} / \mathrm{cm}^{3}$, the crystals would have had to retain approximately 12 percent of xylene. This is at best extremely unlikely. These calculations assume additivity of volumes. If the volume of the crystals and xylene were slightly less than the sum of the separate volumes, the effect on the density would be even smaller.

A separate test showed that no observable amount of polymer remained dissolved in the supernatant xylene, so it was not necessary to make a correction for this.

\section{Calculations}

If we let $P=$ wt of empty pycnometer, $W=$ wt of pycnometer filled with water, $X=$ wt of pycnometer filled with xylene, $T=$ wt of pycnometer filled with mixture of xylene and crystals, and $D=$ wt of pycnometer and dried crystals, the density of the crystals, $\rho_{c}$, can be expressed in terms of the density of water, $\rho_{w}$, by:

$$
\rho_{c}=\frac{\rho_{w}(X-P)(D-P)}{(W-P)(X-P-T+D)} .
$$

The uncertainty in a density determination may be estimated from the uncertainties in the weights by taking the total differential of $\rho_{c}$ in eq (1). We assume that the uncertainty in the "dry" weights $P$ and $D$ is $\pm 0.0002 \mathrm{~g}$. In the case of $W, X$, and $T$ an error of $\pm 0.01 \mathrm{~cm}$ in filling the pycnometer to the line would produce an additional uncertainty of $\pm 0.0003 \mathrm{~g}$, so the total uncertainty for these weights is taken as $\pm 0.0005 \mathrm{~g}$. Using typical values of the weights themselves with these estimates gives an uncertainty in the density of $\pm 0.0040 \mathrm{~g} / \mathrm{cm}^{3}$, which is not far from the standard deviations actually observed.

The uncertainty in the density varies approximately inversely with the amount of crystals used. The uncertainty is caused chiefly by errors in $X$ and $T$. A difference between the density of the xylene used in taking weight $X$ and that used for $T$ could produce a difference several orders of magnitude greater in the calculated density of the crystals for the amount of crystals used here $(0.2$ to $0.4 \mathrm{~g})$. The need for fairly large amounts of crystals was the principal reason for using solutions of the relatively high concentration of 0.75 percent rather than a lower concentration, which would have been desirable because of the lower complexity of the crystals formed.

The weights used in eq (1) are the calculated values in vacuum, i.e., the observed weights plus the net buoyant force of the air. In finding the buoyant force the volumes of the materials being weighed are computed from the assumed values of their weights (and densities) and used to carry through the calculations. These new values of weight (and density) are now used and the calculations repeated. This continues until the assumed and calculated values are equal. Fortunately, we are dealing with a small correction to the weight and the process converges rapidly.

With no liquid in the pycnometer, it was simply weighed daily for a week or so, the weight in vacuum calculated each time, and the average of these values used. The standard deviation of an individual weight was about $0.15 \mathrm{mg}$. When this method was applied to the liquid-filled pycnometer, it was found that the weight decreased at a fairly constant rate-a typical value was $0.1 \mathrm{mg}$ per day. Therefore, the weights in vacuum were plotted against the time and a least squares line was fitted to these points. Figure 4 shows typical plots for three of the pycnometers filled with water. The least squares line was extrapolated to zero time to find the weight used in the calculations. The standard error of this estimate was close to $0.1 \mathrm{mg}$. It was found that a reading taken a few hours after removing the pycnometer from the constant temperature bath often differed slightly from the value obtained by extrapolation.

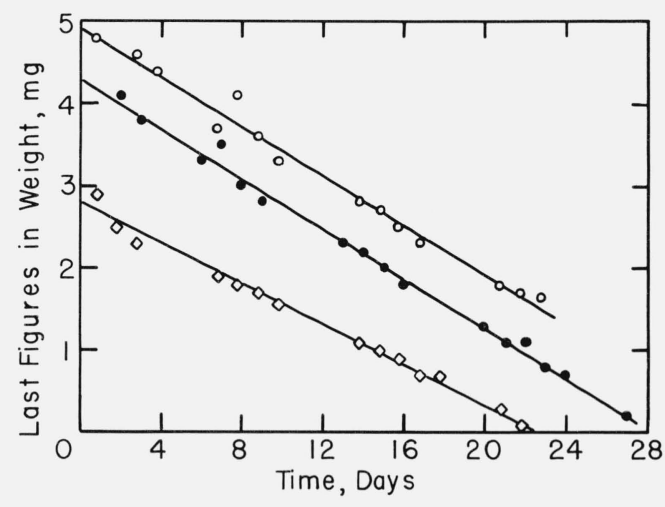

FIGURE 4. Weight loss of pycnometers filled with water. 


\section{Results and Discussion}

The results of eight determinations of the crystal density are given in table 1 . The first series of two was not done in the manner outlined. The crystallization was carried out for $48 \mathrm{hr}$ at $70{ }^{\circ} \mathrm{C}$ rather than 1 week. Ordinary xylene (a mixture of isomers) was distilled and used instead of $p$-xylene. Corrections for the buoyant force of the air were not made, nor was any allowance made for evaporation of liquid from the pycnometer. Attempts to repeat these first two determinations gradually led to the realization that xylene from different bottles varied in density, that the observed weights could change with atmospheric conditions, and that liquid slowly evaporated from the pycnometers no matter how carefully the joints were fitted. Hence, it is felt that the close agreement is not a reliable indication of the accuracy of these two determinations, but may be partly due to the fact that they were run at the same time and partly fortuitous.

The second and third series of measurements were carried out by the method outlined using distilled $p$-xylene, calculating the weights in vacuum, and extrapolating to zero time to determine the weight of the liquid-filled pycnometer. In the second series the needle used to fill the pycnometers did not reach the bottom, so the mixture of xylene and crystals was exposed to a small air gap at the start of the filling process. For the third series a longer needle was used and a little xylene placed in the bottom before adding the mixture, so that there was no exposure to air. The three determinations in the second series were run fairly close to the same time. In the third series \#13 was run first using a mixture that had been stored at room temperature for several months, while \#14 and \#15 were run together using a mixture only a week old.

TABLE 1. Density of polyethylene single crystals grown from solution

\begin{tabular}{|c|c|c|c|c|}
\hline \multicolumn{2}{|c|}{ Pycnometer } & Density & $\begin{array}{l}\text { Avg. density } \\
\text { for series }\end{array}$ & $\begin{array}{l}\text { Std. dev. of an } \\
\text { individual value }\end{array}$ \\
\hline First series & $\begin{array}{l}\# 1 \\
\# 2\end{array}$ & $\begin{array}{r}\mathrm{g} / \mathrm{cm}^{3} \\
0.9637 \\
.9654\end{array}$ & $\begin{array}{l}\mathrm{g} / \mathrm{cm}^{3} \\
0.9645\end{array}$ & $\begin{array}{l}\mathrm{g} / \mathrm{cm}^{3} \\
0.0015\end{array}$ \\
\hline Second series & $\begin{array}{l}\# 14 \\
\# 15 \\
\# 16\end{array}$ & $\begin{array}{l}.9745 \\
.9815 \\
.9746\end{array}$ & .9769 & .0040 \\
\hline Third series & $\begin{array}{l}\# 13 \\
\# 14 \\
\# 15\end{array}$ & $\begin{array}{l}.9885 \\
.9798 \\
.9798\end{array}$ & .9827 & .0050 \\
\hline
\end{tabular}

A Student's " $t$ " test at the 95 percent confidence level shows that the averages for the second and third series are significantly different. A similar test at the 95 percent confidence level indicates that the average for the third series is significantly lower than the crystallographic density, taken as 0.997 $\mathrm{g} / \mathrm{cm}^{3}$. However, it is possible that the density of the crystals increases upon standing at room tem- perature and the higher value for pycnometer \#13 is due to this cause. If pycnometer \#13 is ruled out and the other two values in the third series are combined with the second series, the five readings give an average density of $0.9780 \mathrm{~g} / \mathrm{cm}^{3}$ with a standard deviation of $0.0033 \mathrm{~g} / \mathrm{cm}^{3}$ for an individual reading. Whichever interpretation of the data is made, our result, though slightly higher than the previously quoted values around $0.97 \mathrm{~g} / \mathrm{cm}^{3}$, is significantly less than the crystallographic density.

While it is not our purpose to dwell on the cause of this low density, several possible explanations may be mentioned. The first is that there is an amorphous phase arising perhaps from nonadjacent reentry. This model is difficult to reconcile with the regular crystallographic surfaces usually seen on polyethylene crystals and with the well-known behavior of these crystals upon fracture. Both these pieces of evidence indicate adjacent reentry.

A second possibility is adjacent reentry with a fold length which is not well defined, i.e., an irregular, short-range variation in the fold length of one or two unit cell $c$-dimensions. This is also difficult to reconcile with the regular surfaces of polyethylene crystals. However, it should be pointed out that the experiments which indicate regular surfaces are usually done on crystals grown from very dilute solütion and such crystals may be more perfect than the rather complex ones used in our study. If our crystals had irregular surfaces, with an irregularity of sufficiently short period, then the inability of the solvent to pack regularly on the surface would give spuriously low density results.

A third possible explanation is based on the effect of chain ends. If chain ends are included in the crystals and, as is very likely, if ends of adjacent molecules do not pack in close juxtaposition, an end could cause a large vacancy extending on the average halfway through the thickness of the crystal. This would lead to a density which is dependent on the numberaverage molecular weight. For our polymer such an effect might cause a density deficiency of about 2 percent, just as was observed.

We are deeply indebted to F. Khoury and J. D. Barnes for the optical and electron micrographic observations.

\section{References}

[1] E. W. Fischer and R. Lorenz, Kolloid-L. \& Z. Polymere 189, 97 (1963).

[2] J. B. Jackson, P. J. Flory, and R. Chiang, Trans. Faraday Soc. 59, 1906 (1963)

[3] T. Kawai and A. Keller, Phil. Mag. 8, 1203 (1963)

[4] T. Kawai and A. Keller, Phil. Mag. 8, 1973 (1963)

[5] K. M. Sinnott, J. Polymer Sci., in press.

(Paper 70A3-397) 